Nonl. Analysis and Differential Equations, Vol. 1, 2013, no. 1, 43 - 49 HIKARI Ltd, www.m-hikari.com

\title{
Global Strong Solution to a Nonlinear Dispersive Wave Equation
}

\author{
Yunxi Guo and Ying Wang \\ Department of Applied Mathematics \\ Sichuan University of Science and Engineering \\ 643000, Zigong, China \\ yunxigmaths@163.com
}

Copyright (c) 2013 Yunxi Guo and Ying Wang. This is an open access article distributed under the Creative Commons Attribution License, which permits unrestricted use, distribution, and reproduction in any medium, provided the original work is properly cited.

\begin{abstract}
In this paper, compared with the previous results, a new global existence for strong solutions to the equation is acquired provided that the potential $\left(1-\partial_{x}^{2}\right) u_{0}$ changes sign on $\mathbf{R}$, which improves considerably the previous result.
\end{abstract}

Mathematics Subject Classification: 35G25, 35L05, 35Q35

Keywords: Nonlinear dispersive wave equation; Global existence

\section{Introduction}

In this article, we will consider the nonlinear dispersive wave equation

$$
u_{t}-u_{t x x}+(a+b) u u_{x}-a u_{x} u_{x x}-b u u_{x x x}+\lambda\left(u-u_{x x}\right)=0,
$$

where $a>0, b>0$ and $\lambda \geq 0$ are arbitrary constants, $u$ is the fluid velocity in the $x$ direction, $\lambda\left(u-u_{x x}\right)$ represents the weakly dissipative term.

For $a=2, b=1$ and $\lambda=0$ in Eq.(1), Eq.(1) becomes the famous CamassaHolm equation $[1,2,3]$

$$
u_{t}-u_{t x x}+3 u u_{x}-2 u_{x} u_{x x}-u u_{x x x}=0, \quad t>0, \quad x \in \mathbf{R},
$$


where $u(x, t)$ represents the free surface above a flat bottom. As a model to describe the shallow water motion, Eq.(2) has a bi-Hamiltonian structure and infinite conservation laws and is completely integrable.

For $a=2, b=1$ and $\lambda>0$ in Eq.(1), Eq.(1) changes into weakly dissipative Camassa-Holm equation [4]

$$
u_{t}-u_{t x x}+3 u u_{x}-2 u_{x} u_{x x}-u u_{x x x}+\lambda\left(u-u_{x x}\right)=0, \quad t>0, \quad x \in \mathbf{R} .
$$

For $a=3, b=1$ and $\lambda=0$ in Eq.(1), Eq.(1) becomes the classical Degasperis-Procesi equation $[5,6]$

$$
u_{t}-u_{t x x}+4 u u_{x}-3 u_{x} u_{x x}-u u_{x x x}=0, \quad t>0, \quad x \in \mathbf{R} .
$$

As a model to describe the shallow water wave, Degasperis-Procesi equation (4) also satisfies complete integrablity, bi-Hamiltonian structure, infinite conservation laws and peakon soliton solutions which are analogous to the CamassaHolm. However, they are quite different model(see [5]).

For $a=3, b=1$ and $\lambda>0$ in Eq.(1), Eq.(1) is read as weakly dissipative Degasperis-Procesi equation[7]

$$
u_{t}-u_{t x x}+4 u u_{x}-3 u_{x} u_{x x}-u u_{x x x}+\lambda\left(u-u_{x x}\right)=0, t>0, \quad x \in \mathbf{R} .
$$

In [8], Lai and Wu study global existence and blow-up to Eq.(1) with $\lambda=0$ assumed that the potential $\left(1-\partial_{x}^{2}\right) u_{0}$ does not change sign on $\mathbf{R}$. To our best knowledge, the global strong solution of Eq.(1) under the condition $y_{0} \leq 0$ for $x \leq x_{0}$ and $y_{0} \geq 0$ for $x \geq x_{0}$ seems not have been investigated. Present paper is mainly concerned with global strong solution to Eq.(1) provided that the potential $\left(1-\partial_{x}^{2}\right) u_{0}$ changes sign on R. Since Eq.(1) is a generalization of Camassa-Holm equation and Degasperis-Procesi equation, Eq.(1) loses some important conservation laws that they possess. In the paper, we mainly depend on some useful prior estimates from the equation and the good method presented in Liu and Yin [6] to obtain the blow-up solution for the equation.

\section{Preliminary Notes}

We denote by $*$ the convolution. Note that if $G(x):=\frac{1}{2} e^{-|x|}, x \in \mathbf{R}$, then $\left(1-\partial_{x}^{2}\right)^{-1} f=G * f$ for all $f \in L^{2}(\mathbf{R})$ and $G *\left(u-u_{x x}\right)=u$. Using this identity, the Cauchy problem of Eq.(1) becomes

$$
\left\{\begin{array}{l}
u_{t}+b u u_{x}+\partial_{x} G *\left[\frac{a}{2} u^{2}+\frac{3 b-a}{2}\left(u_{x}\right)^{2}\right]+\lambda u=0, \quad t>0, \quad x \in \mathbf{R}, \\
u(0, x)=u_{0}(x)
\end{array}\right.
$$

which is equivalent to

$$
\left\{\begin{array}{l}
y_{t}+\text { buy }_{x}+\text { ayu }_{x}+\lambda y=0, \quad t>0, \quad x \in \mathbf{R} \\
y=u-u_{x x} \\
u(0, x)=u_{0}(x)
\end{array}\right.
$$


We firstly establish the local well-posedness of solution and blow-up scenario for problem (6).

Lemma 2.1. [9] Given $u_{0} \in H^{s}\left(s>\frac{3}{2}\right)$, there exist a maximal $T=T\left(u_{0}\right)$ and a unique solution $u$ to problem (6), such that

$$
u=u\left(\cdot, u_{0}\right) \in C\left([0, T) ; H^{s}(\mathbf{R})\right) \bigcap C^{1}\left([0, T) ; H^{s-1}(\mathbf{R})\right) .
$$

Lemma 2.2. [9] Let $u_{0} \in H^{s}, s \geq 2$, and $u$ be the corresponding solution to problem (6) with time $T$. Then $T<\infty$ if and only if

$$
\lim _{t \rightarrow T} \inf \left\{\inf _{x \in \mathbf{R}}\left[u_{x}(t, x)\right]\right\}=-\infty
$$

\section{Global strong solution}

In this section, we discuss the global existence of solution to problem (6).

Lemma 3.1. Let $u_{0} \in H^{s}(\mathbf{R})(s>3)$, and $T$ be the maximal existence time of the corresponding solution $u$ to Eq.(1). Then system (18) has a unique solution $q \in C^{1}([0, T) \times \mathbf{R} ; \mathbf{R})$. Moreover, the map $q(t, \cdot)$ is an increasing diffeomorphism of $\mathbf{R}$ with

$$
q_{x}(t, x)=\exp \left(\int_{0}^{t} b u_{x}(s, q(s, x)) d s\right)>0, \quad \forall(t, x) \in[0, T) \times R
$$

and

$$
y(t, q(t, x)) q_{x}^{2}(t, x)=y_{0}(x) \exp \left(\int_{0}^{t}\left[-(a-2 b) u_{x}(s, q(s, x))-\lambda\right] d s\right) .
$$

Proof. From Theorem 2.1, we have $u \in C^{1}\left([0, T) ; H^{s-1}(\mathbf{R})\right)$ and $H^{s-1} \in$ $C^{1}(\mathbf{R})$. We conclude that both functions $u(t, x)$ and $u_{x}(t, x)$ are bounded, Lipschitz in space and $C^{1}$ in time. Applying the existence and uniqueness theorem of ordinary differential equations implies that system (18) has a unique solution $q \in C^{1}([0, T) \times \mathbf{R}, \mathbf{R})$.

Differentiating (18) with respect to $x$ leads to

$$
\left\{\begin{array}{l}
\frac{d}{d t} q_{x}=b u_{x}(t, q) q_{x}, \quad t \in[0, T), \quad b>0 \\
q_{x}(0, x)=1, \quad x \in R,
\end{array}\right.
$$

which yields

$$
q_{x}=\exp \left(\int_{0}^{t} b u_{x}(s, q(s, x)) d s\right) .
$$


For every $T^{\prime}<T$, using the Sobolev embedding theorem gives rise to

$$
\sup _{(s, x) \in\left[0, T^{\prime}\right) \times R}\left|u_{x}(s, x)\right|<\infty .
$$

It is inferred that there exists a constant $K_{0}>0$ such that $q_{x} \geq e^{-K_{0} t}$ for $(t, x) \in[0, T) \times R$.

By computing directly, we derive

$$
\frac{d}{d t}\left[y(t, q(t, x)) q_{x}^{2}(t, x)\right]=-(a-2 b) u_{x}(t, x) y q_{x}^{2}-\lambda y q_{x}^{2}
$$

which results in

$$
y(t, q(t, x)) q_{x}^{2}(t, x)=y_{0}(x) \exp \left(\int_{0}^{t}\left[-(a-2 b) u_{x}(s, q(s, x))-\lambda\right] d s\right) .
$$

The proof of Lemma 3.1 is completed.

Lemma 3.2. Provided that $u_{0} \in H^{s}(\mathbf{R}) \cap L^{1}(\mathbf{R}), s \geq \frac{3}{2}$, if $y_{0}=u_{0}-u_{0 x x}$ satisfies

$y_{0} \leq 0$ for $x \leq x_{0}, y_{0} \geq 0$ for $x \geq x_{0}$ and $y(t, x)=u(t, x)-u_{x x}(t, x)$, then, for $t \in \mathbf{R}_{+}$, it holds

(i) $\|u\|_{L^{1}} \leq e^{-\lambda t}\left\|u_{0}\right\|_{L^{1}}$ on $\mathbf{R}$,

(ii) $\|u\|_{H^{1}}^{2} \leq\left\|u_{0}\right\|_{H^{1}}^{2} \exp \left(-2 \lambda t+\frac{|a-2 b|}{\lambda}\left\|u_{0}\right\|_{L^{1}}\left(1-e^{-\lambda t}\right)\right)$.

Proof. Since $q(t, x)$ is an increasing diffeomorphism of $R$ with $q_{x}(t, x)>0$ with respect to time $t$. We deduce from the assumption that for $t \in[0, T)$,

$$
\begin{cases}y(t, x) \leq 0, & \text { if } \quad x \leq q\left(t, x_{0}\right) \\ y(t, x) \geq 0, & \text { if } \quad x \geq q\left(t, x_{0}\right)\end{cases}
$$

and $y\left(t, q\left(t, x_{0}\right)\right)=0$.

Integrating the first equation of problem (6) with respect to $x$ in interval $\left(-\infty, q\left(t, x_{0}\right)\right]$ yields

$$
\begin{aligned}
\frac{d}{d t} \int_{-\infty}^{q\left(t, x_{0}\right)} u d x=- & b \int_{-\infty}^{q\left(t, x_{0}\right)} u u_{x} d x-\int_{-\infty}^{q\left(t, x_{0}\right)} \partial_{x}\left(G *\left[\frac{a}{2} u^{2}+\frac{3 b-a}{2}\left(u_{x}\right)^{2}\right]\right) d x \\
& -\lambda \int_{-\infty}^{q\left(t, x_{0}\right)} u d x
\end{aligned}
$$

On the other hand, integrating the first equation of problem (6) with respect to $x$ in interval $\left[q\left(t, x_{0}\right),+\infty\right)$ leads to

$$
\begin{aligned}
\frac{d}{d t} \int_{q\left(t, x_{0}\right)}^{\infty} u d x=- & b \int_{q\left(t, x_{0}\right)}^{\infty} u u_{x} d x-\int_{q\left(t, x_{0}\right)}^{\infty} \partial_{x}\left(G *\left[\frac{a}{2} u^{2}+\frac{3 b-a}{2}\left(u_{x}\right)^{2}\right]\right) d x \\
& -\lambda \int_{q\left(t, x_{0}\right)}^{\infty} u d x
\end{aligned}
$$


Subtracting (12) from (13), one has

$$
\begin{gathered}
\left.\frac{d}{d t}\left(\int_{q\left(t, x_{0}\right)}^{\infty} u d x-\int_{-\infty}^{q\left(t, x_{0}\right)} u d x\right)=2 G *\left[\frac{a}{2} u^{2}+\frac{3 b-a}{2}\left(u_{x}\right)^{2}\right]\right)\left.\right|_{x=q\left(t, x_{0}\right)} \\
\left.b u^{2}\right|_{x=q\left(t, x_{0}\right)}-\lambda\left(\int_{q\left(t, x_{0}\right)}^{\infty} u d x-\int_{-\infty}^{q\left(t, x_{0}\right)} u d x\right),
\end{gathered}
$$

which results in

$$
\frac{d}{d t} \int_{-\infty}^{\infty}|u| d x \leq-\lambda \int_{-\infty}^{\infty}|u| d x
$$

Hence, we get

$$
\|u\|_{L^{1}} \leq e^{-\lambda t}\left\|u_{0}\right\|_{L^{1}}
$$

This proves (i).

Using $u=G * y$, one has

$$
u(t, x)=\frac{e^{-x}}{2} \int_{-\infty}^{x} e^{\xi} y(\xi) d \xi+\frac{e^{x}}{2} \int_{x}^{\infty} e^{-\xi} y(\xi) d \xi
$$

from which we obtain

$$
u_{x}(t, x)=-\frac{e^{-x}}{2} \int_{-\infty}^{x} e^{\xi} y(\xi) d \xi+\frac{e^{x}}{2} \int_{x}^{\infty} e^{-\xi} y(\xi) d \xi
$$

from (14), (15) and (11), it follows that

$$
u(t, x)+u_{x}(t, x)=e^{x} \int_{x}^{+\infty} e^{-\xi} y(\xi) d \xi \geq 0, \quad \text { if } \quad x \geq q\left(t, x_{0}\right)
$$

and

$$
u(t, x)-u_{x}(t, x)=e^{-x} \int_{-\infty}^{x} e^{\xi} y(\xi) d \xi \leq 0, \quad \text { if } \quad x \leq q\left(t, x_{0}\right)
$$

From (16) and (17), we get $-u_{x} \leq|u|$ on $\mathbf{R}$.

Multiplying Eq.(1) by $u$ and integrating by parts, we have

$$
\begin{aligned}
\frac{1}{2} \frac{d}{d t} \int_{R}\left(u^{2}+u_{x}^{2}\right) d x & =-\lambda \int_{R}\left(u^{2}+u_{x}^{2}\right) d x-a \int_{R} u u_{x} u_{x x} d x-b \int_{R} u^{2} u_{x x x} d x \\
& =-\lambda \int_{R}\left(u^{2}+u_{x}^{2}\right) d x-\frac{2 b-a}{2} \int_{R} u_{x}^{3} d x \\
& \leq-\lambda \int_{R}\left(u^{2}+u_{x}^{2}\right) d x+\frac{|a-2 b|}{2}\|u\|_{L^{1}}\|u\|_{H^{1}}^{2} \\
& \leq-\lambda \int_{R}\left(u^{2}+u_{x}^{2}\right) d x+\frac{|a-2 b|}{2} e^{-\lambda t}\left\|u_{0}\right\|_{L^{1}}\|u\|_{H^{1}}^{2} .
\end{aligned}
$$


Applying the Gronwall's inequality, one has

$$
\|u\|_{H^{1}}^{2} \leq\left\|u_{0}\right\|_{H^{1}}^{2} \exp \left(-2 \lambda t+\frac{|a-2 b|}{\lambda}\left\|u_{0}\right\|_{L^{1}}\left(1-e^{-\lambda t}\right)\right) .
$$

This completes the proof of Lemma 3.2.

Theorem 3.3. Let $u_{0} \in H^{s}(\mathbf{R}) \cap L^{1}(\mathbf{R}), s>\frac{3}{2}$ and $y_{0}=u_{0}-u_{0 x x}$ satisfies

$$
y_{0} \leq 0 \quad \text { on } \quad\left(-\infty, x_{0}\right] \text { and } y_{0} \geq 0 \quad\left[x_{0}, \infty\right)
$$

for some point $x_{0} \in \mathbf{R}$. Then problem (6) has a global strong solution satisfying

$$
u=u\left(\cdot, u_{0}\right) \in C\left([0, \infty) ; H^{s}(\mathbf{R})\right) \bigcap C^{1}\left([0, \infty) ; H^{s-1}(\mathbf{R})\right) .
$$

Remark. If $x_{0}$ goes to $\pm \infty$, when $\lambda=0$ in Eq.(1), we recover the Theorem 3 obtained by Lai and $\mathrm{Wu}$ [8], where the assumption $y_{0} \geq 0$ on $\mathbf{R}$ or $y_{0} \leq 0$ on $\mathbf{R}$ is needed. Therefore, Theorem 3 in [8] is a special case of Theorem 3.3.

Before proving the theorem, we firstly consider the differential equation

$$
\left\{\begin{array}{l}
q_{t}=b u(t, q), \quad t \in[0, T), \quad x \in \mathbf{R}, \\
q(0, x)=x, \quad x \in \mathbf{R},
\end{array}\right.
$$

where $u$ solves Eq.(1) and $T>0$.

Proof. Here, we assume $s=3$ to prove the theorem. Let $T$ be the maximal time of existence of the solution $u$ to problem (6) with initial data $u_{0} \in H^{3}$.

From (11) and the formulas (14)-(15), we deduce that

$$
\begin{aligned}
u_{x}(t, x) & =-u(t, x)+e^{x} \int_{x}^{\infty} e^{-\xi} y(t, \xi) d \xi \\
& \geq-u(t, x), \quad x \geq q\left(t, x_{0}\right)
\end{aligned}
$$

and

$$
\begin{aligned}
u_{x}(t, x)= & u(t, x)-e^{-x} \int_{-\infty}^{x} e^{\xi} y(t, \xi) d \xi \\
& \geq u(t, x), \quad x \leq q\left(t, x_{0}\right) .
\end{aligned}
$$

Therefore, we have

$$
u_{x}(t, x) \geq-\|u(t, x)\|_{L^{\infty}}, \quad(t, x) \in[0, T) \times \mathbf{R} .
$$

From the Sobolev imbedding theorem and Lemma 3.2, we get

$$
\begin{aligned}
\| u(t, x) & \left\|_{L^{\infty}}^{2} \leq\right\| u(t, x) \|_{H^{1}}^{2} \\
& \leq\left\|u_{0}\right\|_{H^{1}}^{2} \exp \left(-2 \lambda t+\frac{|a-2 b|}{\lambda}\left\|u_{0}\right\|_{L^{1}}\left(1-e^{-\lambda t}\right)\right) .
\end{aligned}
$$


It follows from (21)-(22) that

$$
\begin{aligned}
u_{x}(t, x) \geq & -\|u(t, x)\|_{L^{\infty}} \\
& \geq-\left\|u_{0}\right\|_{H^{1}} \exp \left(-\lambda t+\frac{|a-2 b|}{2 \lambda}\left\|u_{0}\right\|_{L^{1}}\left(1-e^{-\lambda t}\right)\right)
\end{aligned}
$$

where $(t, x) \in[0, T) \times \mathbf{R}$. The above inequality (23) and Theorem 2.2 imply that $T=\infty$. This proves that the solution $u(t, x)$ exists globally in time.

\section{ACKNOWLEDGEMENTS.}

This work was supported by the Research Foundation of SUSE (No.2011KY12) and (No.2011RC10) and the project (No.12ZB080)

\section{References}

[1] R. Camassa and D. Holm, An integrable shallow water equation with peaked solutions, Phys. Rev. Lett., 71( 1993), 1661-1664.

[2] Y. Li and P. Olver, Well-posedness and blow solutions for an integrable nonlinearly dispersive model wave equation, J. Differential Equations,162 (2000), 27-63.

[3] A. Constantin, Existence of permanent and breaking waves for a shallow water wave equation: A geometric approach, Ann. Inst. Fourier (Grenoble), 50 (2000), 321-362.

[4] S. Wu and Z. Yin, Global existence and blow-up phenomena for the weakly dissipative Camassa-Holm equation, J. Differential equations, 246 ( 2009 ), 4309-4321.

[5] J. Escher, Y. Liu and Z. Yin, Global weak solutions and blow-up structure for the Degasperis-Procesi equation, J. Funct. Anal., 241 (2006), 457-485.

[6] Y. Liu and Z. Yin, Global existence and blow-up phnomena for Degasperis-Procesi equation, Comm. Math. Phys., 267 (2006), 801-820.

[7] Z. Guo, Some properties of solutions to the weakly dissipative DegasperisProcesi equation, J.Differential Equations, 246 (2009), 4332-4344.

[8] S. Lai and Y. Wu, Global solutions and blow-up phenomena to a shallow water equation, J. Differential Equations, 249( 2010), 693-706.

[9] Y. Wang, Y. Guo, The Cauchy problem to a shallow water wave equation with a weakly dissipative term, Abstract and Applied Analysis, doi:10.1155/2012/840919.

\section{Received: November, 2012}

